\title{
Relapse of Prior Malignancy
}

National Cancer Institute

\section{Source}

National Cancer Institute. Relapse of Prior Malignancy. NCI Thesaurus. Code C125202.

Clinical and/or laboratory evidence of reemergence of a malignancy after a period of remission. 